\title{
Intrinsic terminators in Mycoplasma hyopneumoniae transcription
}

\author{
Tiago Ebert Fritsch ${ }^{1}$, Franciele Maboni Siqueira ${ }^{1}$ and Irene Silveira Schrank $k^{1,2^{*}}$
}

\begin{abstract}
Background: Mycoplasma hyopneumoniae, an important pathogen of swine, exhibits a low guanine and cytosine (GC) content genome. M. hyopneumoniae genome is organised in long transcriptional units and promoter sequences have been mapped upstream of all transcription units. These analysis provided insights into the gene organisation and transcription initiation at the genome scale. However, the presence of transcriptional terminator sequences in the $M$. hyopneumoniae genome is poorly understood.

Results: In silico analyses demonstrated the presence of putative terminators in $82 \%$ of the 33 monocistronic units (mCs) and in 74\% of the 116 polycistronic units (pCs) considering different classes of terminators. The functional activity of 23 intrinsic terminators was confirmed by RT-PCR and qPCR. Analysis of all terminators found by three software algorithms, combined with experimental results, allowed us to propose a pattern of RNA hairpin formation during the termination process and to predict the location of terminators in the M. hyopneumoniae genome sequence.

Conclusions: The stem-loop structures of intrinsic terminators of mycoplasma diverge from the pattern of terminators found in other bacteria due the low content of guanine and cytosine. In M. hyopneumoniae, transcription can end after a transcriptional unit and before its terminator sequence and can also continue past the terminator sequence with RNA polymerases gradually releasing the RNA.
\end{abstract}

Keywords: Mycoplasma hyopneumoniae, Transcription termination, Intrinsic terminator, Transcriptional units

\section{Background}

Transcription is a highly regulated multi-step process roughly divided into initiation, elongation and termination. Prokaryotic transcription termination can occur via factor-dependent or factor-independent termination and is known to play key roles in regulating genetic systems. Factor-independent termination occurs at defined sequence regions known as intrinsic terminators and factor-dependent termination relies on the destabilisation of transcription complexes by a Rho regulatory protein [1]. Sequence features within intrinsic terminators have been studied in different organisms resulting in models for how these motifs contribute to overall termination efficiency, and have been used to develop

\footnotetext{
* Correspondence: irene@cbiot.ufrgs.br

${ }^{1}$ Centro de Biotecnologia, Programa de Pós-Graduação em Biologia Celular e Molecular, Universidade Federal do Rio Grande do Sul (UFRGS), Porto Alegre, RS, Brazil

${ }^{2}$ Departamento de Biologia Molecular e Biotecnologia, Centro de

Biotecnologia, Universidade Federal do Rio Grande do Sul (UFRGS), Av. Bento Gonçalves 9500, P. 43421, C.P. 15005, Porto Alegre, RS CEP 91501-970, Brazil
}

computational methods to identify terminator elements within genome sequences [2-4].

Mycoplasmas are cell wall-less prokaryotes of the class Mollicutes, phylogenetically related to Gram-positive bacteria and characterised by having small genomes with a low GC content. Mycoplasma hyopneumoniae is considered the etiological agent of porcine enzootic pneumonia, a disease with global distribution and causing significant economic losses in the pig farming industry [5].

In recent years, the genomes of many Mycoplasma species have been completely sequenced, including those of some M. hyopneumoniae strains [6-8]. The sequencing of several mycoplasma genomes has provided an extensive comparative analysis of gene content among different species and information related to gene organisation in large transcriptional units (TUs) [8-10]. Moreover, promoter sequences have been defined and mapped for the $M$. hyopneumoniae genome [11,12] and recently, 
transcriptome analysis has validated the organization of $M$. hyopneumoniae genome in long transcriptional units [10].

However, despite the genome-scale sequencing efforts, prediction and recognition of mycoplasma terminator elements is poorly known. The unusually low GC content of intergenic regions (IRs) in the mycoplasma genome [13] may be the main reason for the lack of success in application of existing computational methods developed to predict putative intrinsic terminators. Moreover, gene coding for Rho regulatory protein was not found in mycoplasma genomes suggesting the absence of factor-dependent transcription termination in these organisms [4].

Studies investigating the presence of intrinsic terminators in mycoplasma were initially contradictory. Analysis of average RNA folding energy near stop codons demonstrated that no stem-loops were formed in Mycoplasma genitalium or Mycoplasma pneumoniae coding sequences (CDS), indicating the existence of qualitatively different and uncharacterised mechanisms for transcription termination [14]. However, the development of algorithms to predict terminators in low GC content genomes allowed the identification of stem-loop structures in some positions of mycoplasma genomes [3,15-17]. Furthermore, several studies showed that intrinsic terminators have a functional role in mycoplasmas [18-20] suggesting that the intrinsic terminators could be the main mode of termination.

Previously, we have described the genome organisation profile and mapped promoter sequences upstream of all transcription units of the $M$. hyopneumoniae genome $[9,11,12]$. These results provided insights into the gene organisation and transcription initiation at the genome scale. To further understand the mechanism of transcription in M. hyopneumoniae genome, in the current study we have analysed and predicted the presence of terminator sequences downstream of the transcription units. We have also determined the role of these predicted sequences, revealing that intrinsic terminators are the main mechanism of transcription termination in $M$. hyopneumoniae.

\section{Methods}

\section{In silico analysis of terminators}

The prediction of terminator sequences was performed in M. hyopneumoniae 7448 (NC_007332) using three software algorithms: TransTermHP [16], WebGesTer [17] and ARNold [21]. The software WebGesTer restricts the search for palindrome sequences in a region ranging from -20 to +270 bp from the CDS stop codon and allows the search of non-canonical terminators (without the U-tract). TransTermHP algorithm restricts the search of palindrome sequences to regions in which occurs the presence of at least three thymines in sequence and, therefore, unable to find non-canonical terminators. ARNold combines two algorithms, Erpin and
RNAmotif, which are based on terminators of Escherichia coli and Bacillus subtilis and do not consider the gene context in which the terminators occur. Figure 1 shows the workflow of our terminator prediction classification. Class 1 terminators $\left(t_{c 1}\right)$ were defined using genome localisation parameters as follows: terminator sequences located at the 3 ' end of the last gene of the polycistronic unit $(\mathrm{pC})$; terminator sequences located at the 3' end of the monocistronic unit $(\mathrm{mC})$; terminator sequences located at the 3 ' end of genes within the pCs. For subsequent analyses steps we have selected all terminator sequences located at the 3 ' end of $\mathrm{pCs}$ or $\mathrm{mCs}$ and established new criteria for terminator classification: i) class 2 terminator $\left(t_{c 2}\right)$ sequences were considered when positioned at the end of $\mathrm{pC}$ or $\mathrm{mC}$ and predicted by at least two different algorithms; ii) class 3 terminators $\left(t_{c 3}\right)$ classification considered all $t_{c 1}$ with two additional features: first the distance between the terminator first nucleotide and the stop codon of the target gene should range from -11 to 200 base pairs and second, the values of Gibbs free energy $(\Delta G)$ should be less than $-4 \mathrm{kcal} / \mathrm{mol}$; and iii) class 4 terminators $\left(t_{c 4}\right)$ were all $t_{c 1}$ that contained only one of the features of class 3 terminators. The terminators in the M. hyopneumoniae genome were mapped using Artemis software [22].

\section{Primer design}

Specific RT-PCR and qPCR primers were designed (based on the M. hyopneumoniae genome sequence GenBank access: NC_007332) to target transcription before and after the selected predicted position of the terminators.

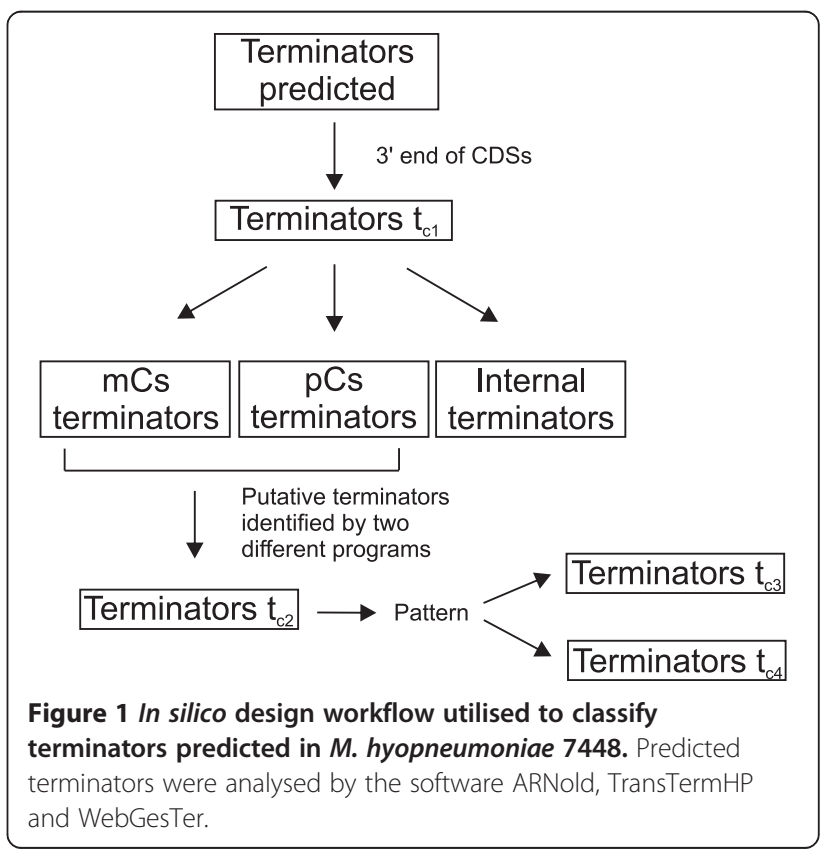


Figure 2 illustrates an example (M. hyopneumoniae 7448 TU_10) of the position of primer pairs to validate the putative terminator. Figure $2 \mathrm{~A}$ shows a global view of the putative terminator $\mathrm{w} \_014$ and Figure 2B demonstrates a detailed position of the primers with the product length expected. As shown in Figure 2B we designed two pairs of primers for each terminator analysis for both RT-PCR and qPCR methodologies. Briefly, the forward primers were positioned inside the coding region of the selected CDS (named Te.F and Ex.F or Ge.F when the primer forward was the same for the two reactions). One reverse primer was positioned between the stop codon of the CDS and the start of the putative terminator (upstream terminator - Te.R). The second reverse primer was positioned immediately after the putative terminator (downstream terminator - Ex.R). A total of 60 primer pairs were prepared for confirmation of 28 putative terminators (Additional file 1). Primers were designed in Vector NTI Advance 10 (Invitrogen, USA).

\section{Culture conditions and RNA isolation}

M. hyopneumoniae strain 7448 isolated from an infected swine (Embrapa, Santa Catarina, Brazil) [6] was grown in $25 \mathrm{ml}$ of Friis broth [23] at $37^{\circ} \mathrm{C}$ for $24 \mathrm{~h}$ with gentle agitation in a roller drum. Total RNA was isolated with RNeasy Mini Kit (Qiagen, USA). For cell lyses, $0.7 \mathrm{ml}$ of RNeasy Lysis Buffer (RLT buffer) in the presence of $0.134 \mathrm{M}$ of $\beta$-mercaptoethanol was used per cultivation flask. The purification was performed according to the manufacturer's instructions, with on-column DNaseI digestion using the RNase-Free DNase Set (Qiagen, Germany) and a second round of treatment with DNase I (Fermentas, USA). DNA absence was monitored to below PCRdetectable levels. Extracted RNA was analysed by gel electrophoresis and quantified in the Qubit system (Invitrogen, USA). Finally, RNA quality and integrity were determined by the evaluation of the RNA Integrity Number (RIN) using the Agilent 2100 Bioanalyzer (Agilent, USA). Values equal to or greater than 9.5 indicated sufficient quality.

\section{Reverse transcriptase PCR (RT-PCR) assay}

For first-strand cDNA synthesis we used $1 \mu \mathrm{g}$ of total RNA, 10 pmol of specific reverse primer (Additional file 1) and $10 \mathrm{mM}$ of deoxynucleotide triphosphates. The mixture was heated to $70^{\circ} \mathrm{C}$ for $5 \mathrm{~min}$ and then incubated on ice for $5 \mathrm{~min}$. First-strand buffer (Promega,

A

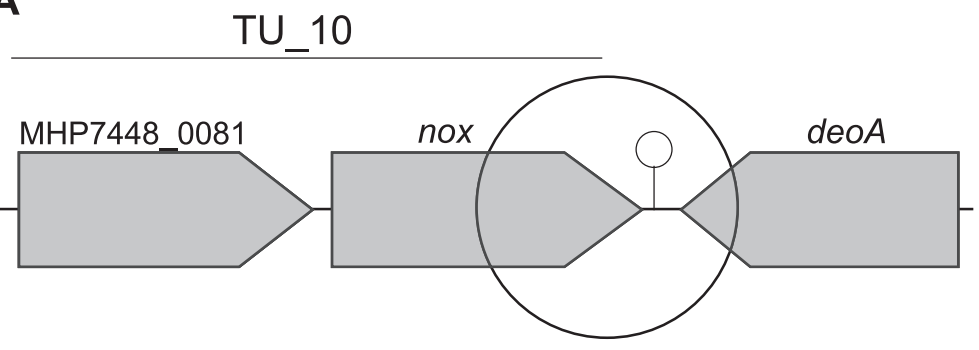

B

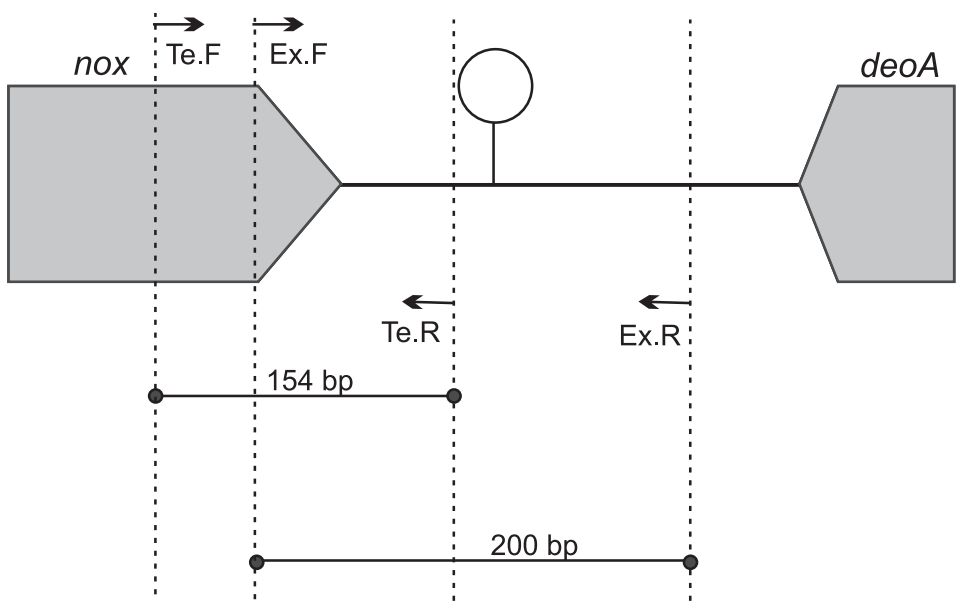

Figure 2 Schematic representation of primers design for experimental analyses by RT-PCR or qPCR. A: Representation of polycistronic unit 10 with the terminator W_014 (hairpin). B: Enlargement of highlighted region in A, showing the position of primers for terminator validation. Black arrows Te.F, Te.R, Ex.F and Ex.R represent the primers U.10.Te.F, U.10.Te.R, U.10.Ex.F and U.10.Ex.R, respectively. (U.10 - polycistronic unit 10, Te - terminator, Ex - external, $F$ - forward and $R$ - reverse). 
USA), $0.1 \mathrm{M}$ dithiothreitol, $40 \mathrm{U}$ RNase inhibitor (Promega, USA) and 200 U M-MLV RT (Moloney Murine Leukemia Virus Reverse Transcriptase-Promega, USA) were then added to a total volume of $20 \mu \mathrm{l}$. The reaction was incubated at $37^{\circ} \mathrm{C}$ for $60 \mathrm{~min}$ followed by $15 \mathrm{~min}$ at $70^{\circ} \mathrm{C}$ for enzyme inactivation. A negative control was prepared in parallel, differing only by the absence of the RT enzyme.

PCRs included $1 \mathrm{U}$ GoTaq DNA polymerase (Promega, USA), 5X of GoTaq buffer, $1 \mathrm{mM}$ of each deoxynucleotide triphosphate, 10 pmol of each primer (Additional file 1) and $1 \mu \mathrm{l}$ of the first-strand cDNA reaction in a final volume of $25 \mu \mathrm{l}$. A negative control of RT-PCR was prepared in parallel, which differed only by the absence of cDNA, and no genomic DNA was added to the reaction mixture for the PCR negative control. A PCR positive control was prepared using the genomic DNA of $M$. hyopneumoniae 7448 as the template. The PCR conditions were: 1 cycle at $94^{\circ} \mathrm{C}$ for 5 min followed by 30 cycles of $94^{\circ} \mathrm{C}$ for $30 \mathrm{~s}$; denaturation and extension temperature and time varied according to each primer pair (Additional file 1). The final extension step was at $72^{\circ} \mathrm{C}$ for $10 \mathrm{~min}$. Reaction products were analysed in $1.2 \%$ agarose gels.

\section{qPCR experimental design}

Quantitative RT-PCR was performed using 1:50 cDNA prepared as described for RT-PCR as template and Platinum SYBR Green qPCR SuperMix-UDG (Invitrogen, USA) on the StepOne Real-Time PCR System (Applied Biosystems, USA). The qPCR reactions were carried out at $90^{\circ} \mathrm{C}$ for $2 \mathrm{~min}$ and $95^{\circ} \mathrm{C}$ for $10 \mathrm{~min}$ followed by 40 cycles of $95^{\circ} \mathrm{C}$ for $15 \mathrm{~s}$ and $55^{\circ} \mathrm{C}$ for $1 \mathrm{~min}$ each. The specificity of the synthesised products and the absence of primer dimers were visualised using a melting curve analysis for each reaction. Amplification efficiency for each primer pair was calculated using the LinRegPCR software application [24] and the mean efficiency values for each primer were added to Additional file 1. This efficiency value was used for the quantification analysis.

Relative expression of mRNA was calculated by the $2^{-\Delta \mathrm{Ct}}$ method [25]. To control for all the experiments the threshold cycle (CT) values were normalised to the reference gene MHP7448_0333 [12,26]. The CT of each test target represents the average of three reactions. Three independent biological replicates were done for each target gene. We performed statistical analysis using GraphPad Prism 6 software. A two-tailed unpaired $t$-test was used to test for differences in the relative expression values between the regions before and after the terminator structure $(\mathrm{P}<0.05)$.

\section{Results}

\section{Prediction of terminator sequences}

Computational analysis of $M$. hyopneumoniae genome using three different algorithms predicted 1068 terminators: 439 terminators using ARNold software, 334 using
TransTermHP software and 295 using WebGesTer software (Table 1 and Additional file 2). These terminators were named using the initial letter of the software $(\mathrm{a}=\mathrm{AR}$ Nold, $\mathrm{t}=$ TransTermHP and $\mathrm{w}=$ WebGesTer) followed by a number. For example terminator t_001 is the first terminator predicted by TransTermHP software. Terminators named "wf" represent terminators classified as unstable terminators in the WebGesTer software (Additional files 2, 3 and 4).

The classification criteria defined in Figure 1 were applied to validate the predicted terminators. Class 1 terminators, named $t_{\mathrm{c} 1}$, should be positioned at the 3 ' end of the CDS. Using this criterion, 556 terminators of the 1068 predicted terminators were selected (52\%) (Table 1). The remaining predicted terminators were localised within coding regions or on the antisense strand and therefore, were not further analysed in this work.

The $556 \mathrm{t}_{\mathrm{c} 1}$ terminators were analysed according to the genomic context of $M$. hyopneumoniae. The M. hyopneumoniae genome is organised in 33 monocistronic units $(\mathrm{mCs})$ and 116 polycistronic units $(\mathrm{pCs})$ containing two or more ORFs [9]. Therefore, systematic terminator localisation was performed in the downstream regions of all annotated ORFs in the genome. This analysis revealed the presence of $65 t_{c 1}$ terminators at the end of mCs, $255 t_{c 1}$ terminators at the end of pCs and $236 t_{c 1}$ terminators at the 3' end of some ORFs that were located internal in some polycistronic units (identified as internal genes) (Table 1). Moreover, more than one predicted terminator sequence can be found in some transcriptional units. Class 1 terminators were identified in all $33 \mathrm{mCs}$ and in 106 of the $116 \mathrm{pCs}$ (Additional files 3 and 4) suggesting that transcription termination occurs preferentially at the ends of $\mathrm{mCs}$ and $\mathrm{pCs}$.

The class 1 terminators $\left(t_{\mathrm{c} 1}\right)$ were further divided into class 2 , class 3 and class 4 terminators. To ensure the reliability of the generated data, the $\mathrm{mC}$ and $\mathrm{pC} \mathrm{t}_{\mathrm{c} 1}$ terminators located at the same position by at least two algorithms were considered putative terminators and named class 2 terminators $\left(t_{c 2}\right.$; Additional file 5$)$. Using this approach we were able to identify class $t_{\mathrm{c} 2}$ terminators in seven of the $33 \mathrm{mCs}(21 \%)$ and in 39 of the 116 pCs (34\%) (Table 2). Detailed analysis of the characteristics of these terminators revealed the presence of two distinguishing features: i) the distance between the start of the terminator and the stop codon of the target gene showed a range of $-11 \mathrm{bp}$ to $200 \mathrm{bp}$ and ii) the values of Gibbs free energy $(\Delta G)$ were less than $-4 \mathrm{kcal} / \mathrm{mol}$ (Figure 3). The presence of two class $t_{c 2}$ terminators at a distance greater than $230 \mathrm{bp}$ from the stop codon (see Figure 3A) was identified at 3' end of TU_09 (fusA) and TU_114. (MHP7448_r2). However, further analysis revealed the presence of other $\mathrm{t}_{\mathrm{c} 2}$ terminators at the 3 ' end of these two pCs at position 39 or 41 for TU_09 and at positions 167, 185 or 186 for TU_114 (Additional file 2). 
Table 1 Terminators predicted in Mycoplasma hyopneumoniae

\begin{tabular}{|c|c|c|c|c|c|}
\hline & Genic context & WebGesTer & TransTermHP & ARNold & Total \\
\hline Terminators predicted & & 295 & 334 & 439 & 1068 \\
\hline \multirow[t]{4}{*}{ Class 1 terminator $\left(t_{c 1}\right)$} & $\mathrm{pC}$ terminators & 143 & 73 & 39 & 255 \\
\hline & $\mathrm{mC}$ terminators & 40 & 20 & 5 & 65 \\
\hline & Internal terminators & 95 & 99 & 42 & 236 \\
\hline & Total $t_{c 1}$ & 278 & 192 & 86 & 556 \\
\hline
\end{tabular}

To increase the number of $\mathrm{mCs}$ and $\mathrm{pCs}$ containing putative terminators (similar to class 2 terminators) all the remaining $t_{c 1}$ terminators were screened using the features defined in class 2 terminators (see Figure 3). Terminators that satisfied both criteria (distance to stop codon and $\Delta G$ ) were reclassified as class 3 terminators $\left(t_{c 3}\right)$ and those that displayed only one of the two criteria were named class 4 terminators $\left(t_{c 4}\right)$. It is important to point out that, in contrast to class 2 terminators, the class 3 terminators were defined by only one algorithm. Class 3 terminators were identified in 14 of $33 \mathrm{mCs}$ (43\%) and in 36 of $116 \mathrm{pCs}$ (31\%) increasing the number of $\mathrm{mCs}$ and $\mathrm{pCs}$ with putative terminators to 21 (64\%) and 75 (65\%), respectively (Table 2). Class 4 terminators $\left(\mathrm{t}_{\mathrm{c} 4}\right)$ were found in six mCs $(18 \%)$ and eleven pCs $(9 \%)$ of the $M$. hyopneumoniae genome.

\section{Experimental validation of M. hyopneumoniae terminators}

RT-PCR and qPCR were used to test whether the predicted terminators were related to transcription termination in M. hyopneumoniae. The terminator validation hypothesis was based on the following premises: i) $\mathrm{mC}$ or $\mathrm{pC}$ transcripts ended at the predicted terminator sequences; and ii) no $\mathrm{mC}$ or $\mathrm{pC}$ transcripts were present after the predicted terminator sequences. Primer pairs (Figure 2) were positioned to validate this hypothesis, similar to the terminator validation previously used by Arrebola et al. [27].

RT-PCR was performed for 15 putative terminator sequences. Among the putative terminator sequences analysed, four belonged to class $t_{c 2}$, one to class $t_{c 3}$ and 10 to class $t_{\mathrm{c} 4}$ (Table 3 ). The Figure 4 shows the validation of a $t_{c 2}$ terminator $\left(w_{-}\right.$014) in TU_10, used as example, where two primer pairs were designed for RT-

Table 2 Terminator distribution according to the genome organisation of $M$. hyopneumoniae

\begin{tabular}{lll}
\hline Class & $\mathbf{m C}$ & $\mathbf{p C}$ \\
\hline Class $_{\mathrm{c2}}$ & $7(21 \%)$ & $39(34 \%)$ \\
Class $_{\mathrm{c3}}$ & $14(43 \%)$ & $36(31 \%)$ \\
Class $_{\mathrm{c4}}$ & $6(18 \%)$ & $11(9 \%)$ \\
Without terminator $_{\text {Total }}$ & $6(18 \%)$ & $30(26 \%)$ \\
\hline
\end{tabular}

PCR analysis. As shown in Figure 2, primer pairs were positioned to analyse the presence of transcripts upstream and immediately downstream of the predicted terminator. TU_10 is composed of genes MHP7448_0081 and nox. At the end of the nox gene, three $t_{c 2}$ terminators were identified (Additional file 2). The experimental analysis considered the w_014 terminator located at $39 \mathrm{bp}$ of the nox gene stop codon. This terminator is formed of a stem of 11 nucleotides with only one mismatch, one loop of three nucleotides, the U-tract and has a $\Delta G$ of $-7.08 \mathrm{kcal} / \mathrm{mol}$ (Figure 4A). As demonstrated in Figure 4B (lines 3 and 7) amplification was observed only when primers were positioned upstream of the terminator sequence indicating that the w_014 terminator is responsible for transcription termination in M. hyopneumoniae TU_10.

Transcription termination was also demonstrated in the presence of $t_{c 2}$ terminators $t \_264$ and $w_{-}$102 located at TU_89 and TU_113, respectively (Table 3). In six $t_{c 4}$ terminators (wf_491, wf_513, wf_375, wf_137, wf_202 and wf_215), amplification product was detected only when using primers located upstream of the putative terminator sequence, suggesting that the presence of $t_{\mathrm{c} 4}$ terminators at the 3' end of $\mathrm{mC} \_28, \mathrm{mC} \_33, \mathrm{TU} \_60, \mathrm{TU} \_66, \mathrm{TU} \_98$ and TU_103 were responsible for transcription termination (Table 3). These data shows that the putative intrinsic terminators are functional in mycoplasmas.

In the other class 2 terminator (w_076), class 3 terminator (w_053) and four class 4 terminators (wf_204, w_202, wf_075, w_147), amplification products were detected when primers located downstream of the terminator were used and, therefore, could not be validated by RT-PCR. However, it is possible that some terminators, although incapable of efficient transcription termination, could cause a decrease in the level of transcription. To confirm this hypothesis, RNA expression level was quantified using qPCR.

Real-time quantitative PCR was performed for selected class 2, class 3 and class 4 terminators based on the feasibility of primer design, as the M. hyopneumoniae genome has an intergenic low GC content. A total of 16 terminators were analysed by qPCR (Table 4): eight $t_{c 2}$ (Figure 5 ), seven $t_{c 3}$ (Figure 6) and one $t_{c 4}$ terminators (Figure 7). In order to compare RT-PCR and qPCR methodologies, terminator w_102 of TU_113, a t $\mathrm{c}_{\mathrm{c} 2}$ terminator previously confirmed by RT-PCR (Table 3), was also analysed by qPCR (Figure 5 ). 


\section{A Distance to stop codon}

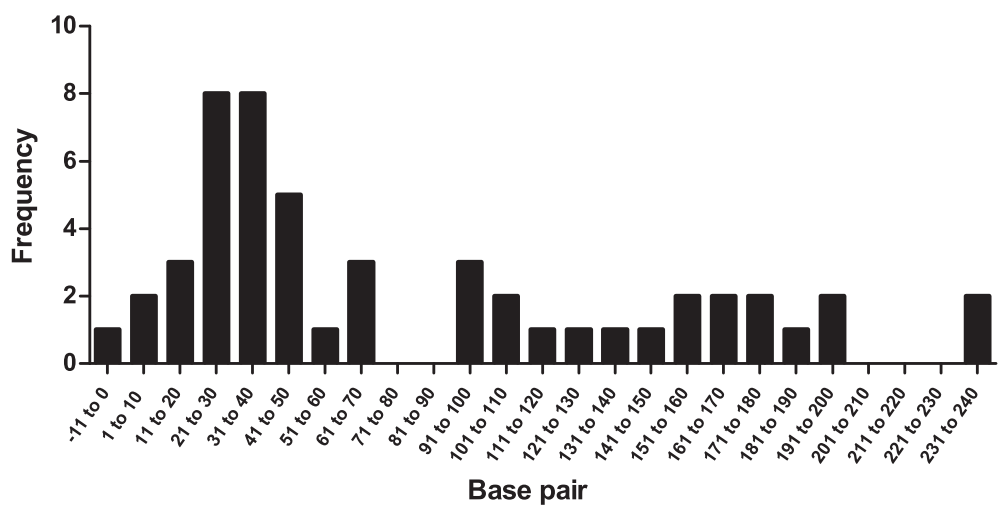

B

Gibbs free energy

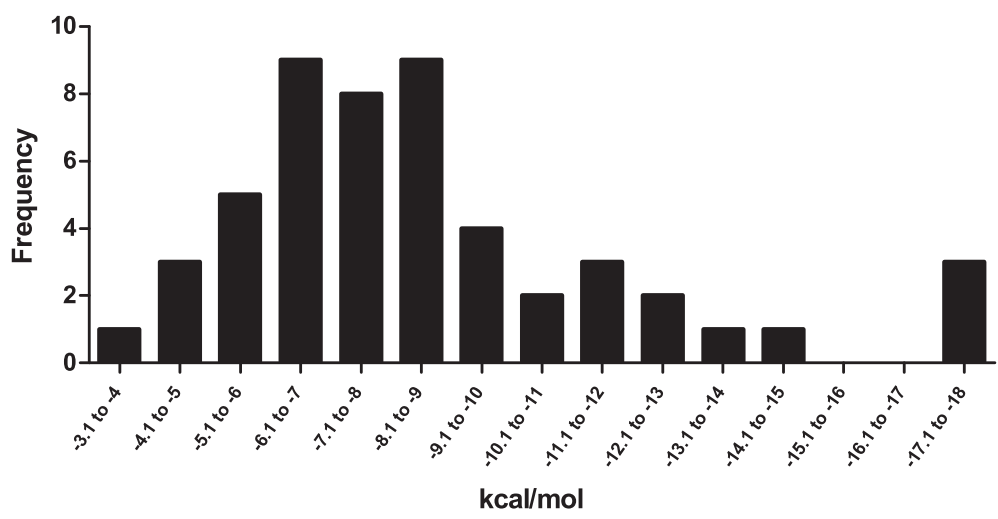

Figure $\mathbf{3}$ Characteristics of class $\mathbf{2}$ terminators. The graphics show the distribution of the class 2 terminators according to distance to stop codon $\mathbf{( A )}$ and the values of Gibbs free energy determined by the algorithm that predicted the terminator (B). Negative distances indicate the overlap with the stop codon of the gene.

For the majority of tested terminators primer efficiency was greater than $80 \%$. Only the primers used to analyse the terminator in TU_62 showed, in both primer pairs, a lower efficiency of $65 \%$ (Additional file 1). Moreover, the primer pairs designed for terminator sequences $\mathrm{w}_{-} 102$ in TU_113 and t_055 in TU_20 revealed differences in efficiency between upstream and downstream primers of around $50 \%$ (Additional file 1). This result is probably related to the absence of detected transcripts in the $\mathrm{qPCR}$ (Figure 5).

The eight class 2 transcriptional terminators analysed by qPCR were localised at 3 ' end of the last gene in TU_09, TU_11, TU_20, TU_58, TU_62, TU_83, TU_115 and TU_113 (Figure 5). Real-time quantitative PCR demonstrated that only transcripts found for TU_09, TU_11, TU_20, TU_58 and TU_113 had similar expression levels to the gene used for normalization (MHP7448_333) in these experiments. The transcriptional terminators were characterised by comparison between transcripts level using primers upstream of the putative terminator sequence (Terminator) and downstream of the terminator sequence (External). Terminators w_109 in TU_09, t_055 in TU_20 and w_102 in TU_113 can be considered valid terminators as no transcripts were detected downstream of the terminator sequence (Figure 5 see External). The other $t_{c 2}$ class terminators, $t \_022$ in TU_11, a_207 in TU_58, t_173 in TU_62, a_310 in TU_83, and w_093 in TU_105 displayed a significant $(0.001<\mathrm{P}<0.05)$ decrease in transcription when downstream primers were used (see Figure 5 Terminator versus External) and also could be related to transcriptional termination.

Transcriptional terminators classified as class 3 (w_016 in mC_04, w_053 in mC_15, w_082 in mC_24, w_140 in TU_31, w_055 in TU_47, wf_140 in TU_68 and w_181 in TU_77) and class 4 (wf_204 in mC_30) terminators were also analysed by qPCR (Figure 6 and Figure 7). Similar to some $t_{\mathrm{c} 2}$ class terminators, all $t_{\mathrm{c} 3}$ and $t_{\mathrm{c} 4}$ class terminators displayed a significant $(0.01<\mathrm{P}<0.05)$ decrease in transcription when downstream primers were used, with exception of w_082 in mC_24 (Figure 6 and Figure 7; see Terminator versus External). Interestingly, a second $t_{c 3}$ (w_083) putative terminator was localised at 74 bp downstream of the first 
Table 3 Predicted terminators analysed by RT-PCR

\begin{tabular}{|c|c|c|c|c|c|c|}
\hline Terminator & Validation $^{1}$ & Localisation & Class & Gene $^{2}$ & Distance $(b p)^{3}$ & $\Delta \mathrm{G}^{4}$ \\
\hline w_053 & No & mC_15 & $t_{c 3}$ & pyrG & 82 & -5.01 \\
\hline wf_491 & TE & mC_28 & $\mathrm{t}_{\mathrm{c4}}$ & $p d h D-1$ & 193 & -4.51 \\
\hline wf_204 & No & mC_30 & $\mathrm{t}_{\mathrm{c4}}$ & pulA & 131 & -2.48 \\
\hline w_202 & No & mC_31 & $\mathrm{t}_{\mathrm{c4}}$ & dam & 195 & -4.69 \\
\hline wf_513 & TE & mC_33 & $t_{c 4}$ & $g c p$ & 190 & -4.36 \\
\hline t_020 & TE & TU_010 & $t_{c 2}$ & nox & 40 & -6.5 \\
\hline wf_075 & No & TU_036 & $\mathrm{t}_{\mathrm{c4}}$ & rpsT & -9 & -3.94 \\
\hline w_147 & No & TU_039 & $t_{c 4}$ & lip & 181 & -4.76 \\
\hline wf_375 & TE & TU_060 & $\mathrm{t}_{\mathrm{c4}}$ & $g / p F$ & 29 & -2.22 \\
\hline wf_137 & TE & TU_066 & $\mathrm{t}_{\mathrm{c4}}$ & metG & 149 & -3.21 \\
\hline w_076 & No & TU_085 & $t_{c 2}$ & $p d h D$ & 37 & -9.01 \\
\hline t_264 & TE & TU_089 & $t_{c 2}$ & tuf & 34 & -7.5 \\
\hline wf_202 & TE & TU_098 & $\mathrm{t}_{\mathrm{c4}}$ & $f p g$ & 52 & -2.53 \\
\hline wf_215 & TE & TU_103 & $\mathrm{t}_{\mathrm{c4}}$ & MHP7448_0621 & 198 & -3.11 \\
\hline w_102 & TE & TU_113 & $t_{c 2}$ & MHP7448_0665 & 43 & -11 \\
\hline
\end{tabular}

${ }^{1}$ Presence of functional activity. TE - transcription termination; TD - transcription decreasing; No - without difference in transcript upstream and downstream of the terminator sequence.

${ }^{2} \mathrm{Gene}$ at the end of the transcription unit.

${ }^{3}$ Distance between stop codon of the gene and the first nucleotide of terminator sequence.

${ }^{4}$ Value of free energy of Gibbs identified by the software that predicted the terminator.

terminator w_082 (Additional file 2). The presence of two putative terminators in this monocistronic unit suggests that they could have complementary functions.

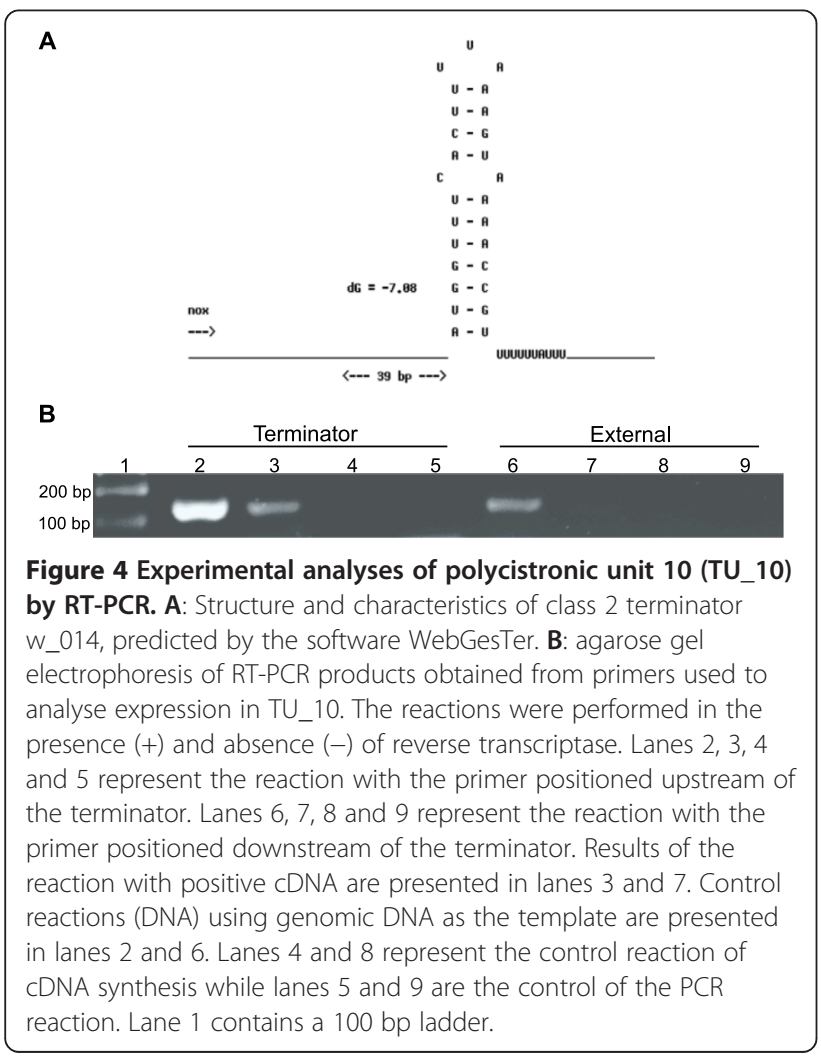

Aiming to summarize our results Figure 8 shows the localisation of both promoter sequences and intrinsic terminators according the genome organisation of $M$. hyopneumoniae. Previous works of our group have demonstrated that the genome is organized preferably in long transcriptional units containing promoter sequences upstream the start codon of the first gene of the units [9,11]. Among the putative class 2 and class 3 terminators, represented in Figure 8, the functional terminators at the end of TU_09, TU_10, TU_11 and mC_04, were also experimentally demonstrated by RT-PCR and qPCR analyses (Tables 3 and 4).

\section{Discussion}

The importance of the characterisation of Rho-independent termination in mycoplasma can be related with the accurate prediction of transcription units in these bacteria. In general, the properties of intrinsic terminators, such as sequence and the structural features of the hairpin and, in some cases, the U-tract of the nascent transcript, could be involved in transcription termination. Previous works have demonstrated the predominance and conservation of Rhoindependent or intrinsic termination among the Mollicutes class $[3,4,16]$.

We identified transcription terminators for M. hyopneumoniae in silico using three algorithms that demonstrated differences in efficiency of prediction. This method allowed the prediction of 1068 terminators in M. hyopneumoniae genome. Moreover, detailed analysis revealed the prediction of at least one terminator sequence in 253 of the 705 M. hyopneumoniae ORFs (35.9\%). 
Table 4 Predicted terminators analysed by qPCR

\begin{tabular}{|c|c|c|c|c|c|c|}
\hline Terminator & Validation ${ }^{1}$ & Localisation & Class & Gene $^{2}$ & Distance $(b p)^{3}$ & $\Delta \mathrm{G}^{4}$ \\
\hline w_016 & TD & mC_04 & $t_{c 3}$ & rbgA & 88 & -6.06 \\
\hline w_053 & TD & mC_15 & $t_{c 3}$ & pyrG & 82 & -5.01 \\
\hline W_082 & No & mC_24 & $t_{c 3}$ & gyrA & 75 & -6.41 \\
\hline wf_204 & $\mathrm{TD}$ & mC_30 & $\mathrm{t}_{\mathrm{c4}}$ & pulA & 131 & -2.48 \\
\hline W_109 & TE & TU_009 & $t_{c 2}$ & fusA & 39 & -17.8 \\
\hline t_022 & $\mathrm{TD}$ & TU_011 & $\mathrm{t}_{\mathrm{c} 2}$ & deoA & 119 & -10 \\
\hline t_055 & $\mathrm{TE}$ & TU_020 & $t_{c 2}$ & $r p m B$ & 49 & -7.6 \\
\hline w_140 & $\mathrm{TD}$ & TU_031 & $t_{c 3}$ & trmE & 35 & -7.81 \\
\hline W_055 & TD & TU_047 & $t_{c 3}$ & ugpQ & 18 & -8.9 \\
\hline a_207 & TD & TU_058 & $t_{c 2}$ & hit & 27 & -5.8 \\
\hline t_173 & $\mathrm{TD}$ & TU_062 & $\mathrm{t}_{\mathrm{c} 2}$ & MHP7448_0372 & 43 & -9.4 \\
\hline wf_140 & $\mathrm{TD}$ & TU_068 & $t_{c 3}$ & ruvB & 42 & -3.91 \\
\hline W_181 & TD & TU_077 & $t_{c 3}$ & $r p / A$ & 107 & -6.19 \\
\hline a_310 & $\mathrm{TD}$ & TU_083 & $t_{c 2}$ & MHP7448_0494 & 24 & -5.12 \\
\hline w_093 & TD & TU_105 & $t_{c 2}$ & MHP7448_0628 & 109 & -8.35 \\
\hline w_102 & TE & TU_113 & $t_{c 2}$ & MHP7448_0665 & 43 & -11 \\
\hline
\end{tabular}

${ }^{1}$ Presence of functional activity. TE - transcription termination; TD - transcription decreasing; No - without difference in transcript upstream and downstream of the terminator sequence.

${ }^{2}$ Gene at the end of transcription unit.

${ }^{3}$ Distance between stop codon of the gene and the first nucleotide of terminator sequence.

${ }^{4}$ Value of free energy of Gibbs identified by the software that predicted the terminator.

In comparison to $E$. coli, the intrinsic terminators found in the M. hyopneumoniae genome display relatively high values of Gibbs free energy density of stemloop formation correlated to the low content of guanine and cytosine in the Mollicutes genome [13]. Similar values were also found in intrinsic terminators of $M$. genitalium and $M$. pneumoniae and are probably responsible for previous difficulties identifying intrinsic terminators in M. pneumoniae based on analysis of average RNA folding energy near stop codons [3,14]. The presence of a U-tract following the stem-loop structure was detected for the majority of the predicted $M$. hyopneumoniae terminators. However, approximately $20 \%$ of M. hyopneumoniae stem-loop formation revealed absence of the U-tract tail of the nascent transcript. These results are similar to those found in other mycoplasma species, suggesting that the U-tract tail is not an essential requisite for intrinsic terminators in this genus [4]. Nevertheless, the efficiency of transcription termination is maintained in intrinsic terminators without the U-tract $[28,29]$.

Detailed localisation analysis of the 1068 predicted terminators allowed us to select only those found at the 3' end of pCs or mCs. Using this approach we were able to distinguish inter- from intracistronic terminator-like structures and select 320 putative Rho-independent terminators at the end of $\mathrm{mCs}$ and $\mathrm{pCs}$. The prediction efficiency was different among the software, reflecting the specific algorithm used for each one. The best results were obtained with the software WebGesTer, as is the only one of the three software packages that takes into consideration the gene context, considering a region ranging from -20 to $+270 \mathrm{bp}$ from the stop codon of CDS, and allows the detection of terminators without a U-tract [17]. Of the 295 terminators predicted by this program 279 were classified as class $t_{c 1}$. The software TransTermHP identified 191 terminators class $t_{c 1}$. The lower number of predicted terminators found by the TransTermHP software is related to the restrict search of palindromic sequences in regions with the presence of at least three thymines in sequence. Therefore, the TransTermHP program is able to found only canonical sequences [16]. The ARNold software use the patterns defined for E. coli and B.subtilis and do not take in consideration the gene context in which the terminator occur [21]. This probably explains why only 86 of the 439 (19\%) predicted terminators, classified as class 1 terminators, were found by this program. The remaining terminators predicted by ARNold program were localised within coding regions or on the antisense strand and therefore were not analysed in this paper.

Class $t_{\mathrm{c} 1}$ terminators were identified in all $33 \mathrm{mCs}$ and in 106 of 116 pCs. This method was unable to localise terminators in only $10(8.6 \%)$ pCs. Interestingly, in seven of them (TU_15, TU_16, TU_52, TU_72, TU_78, TU_104 and TU_106) the last ORF is classified as hypothetical. 


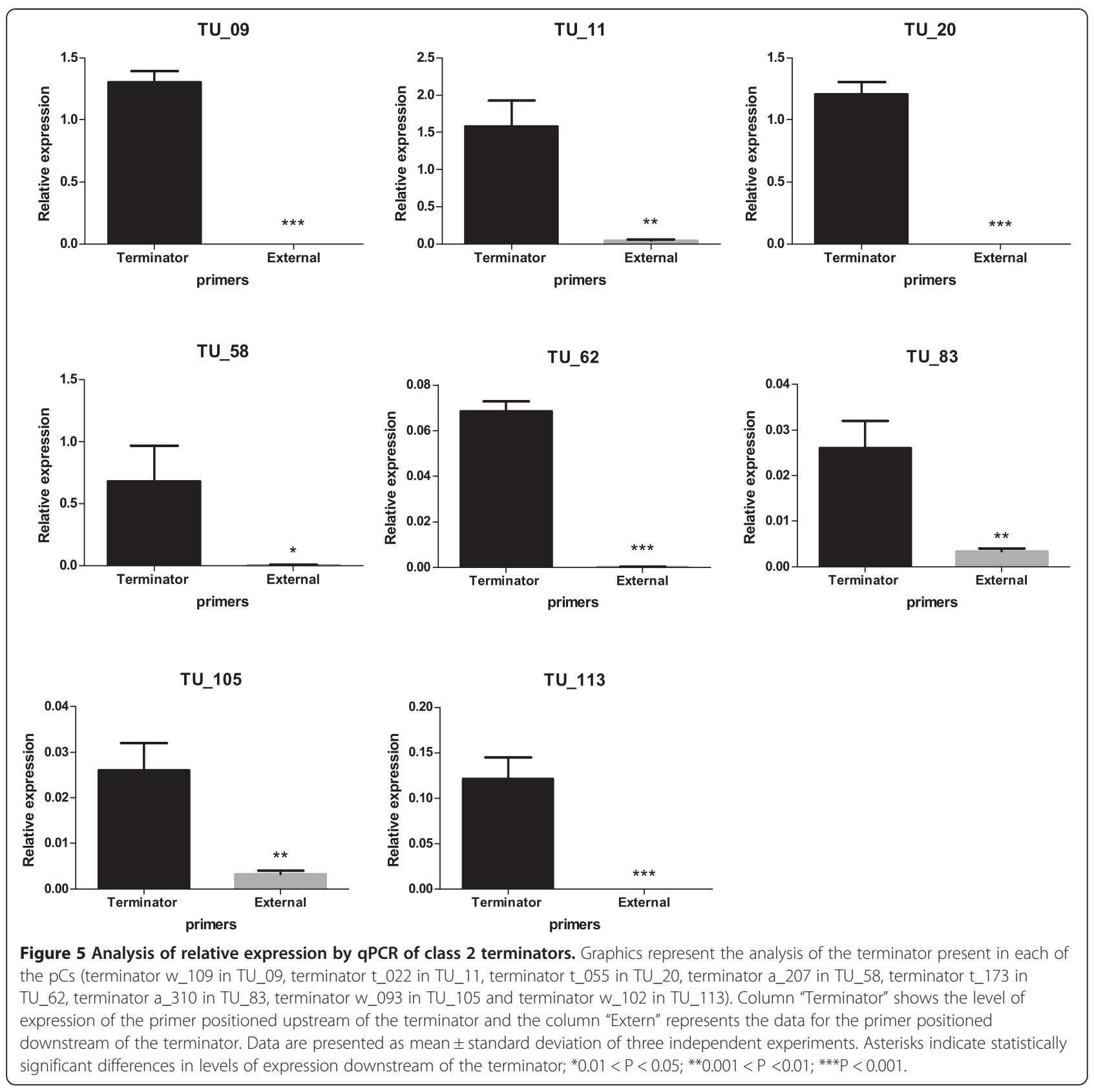

Moreover, terminator sequences were found upstream of the last ORF in TU_15, TU_16, TU_72 and TU_106 suggesting that transcription could be terminated before the last ORF in these polycistronic units. In three pCs (TU_72, TU_104 and TU_115) the presence of a gene similar to one that codes for transposase was detected. Therefore, we suggest that Rho-independent termination occurs at specific sequences in M. hyopneumoniae genome.

Aiming to distinguish $M$. hyopneumoniae Rho-independent terminators from random stem-loop structure sequences we established rules to discover their distinguishing properties. We used class 2 terminator classification $\left(t_{c 2}\right)$ to propose a model of intrinsic terminators and as a decision rule to define as putative terminators in $M$. hyopneumoniae genome. The decision rule had four parameters: i) an inverted repeat in the primary DNA sequence positioned at the 3' end of $\mathrm{pC}$ or $\mathrm{mC}$ followed or not by a short stretch of thymine residues; ii) predicted by at least two different algorithms; iii) distance between the terminator first nucleotide and the stop codon of the target gene range from -11 to $200 \mathrm{bp}$; and iv) the values of Gibbs free energy $(\Delta \mathrm{G})$ should be less than $-4 \mathrm{kcal} / \mathrm{mol}$. However, the application of these four rules validated terminators in silico in only $21 \%$ of the mCs (seven of the 33) and in $34 \%$ of the pCs (39 of the 116). 


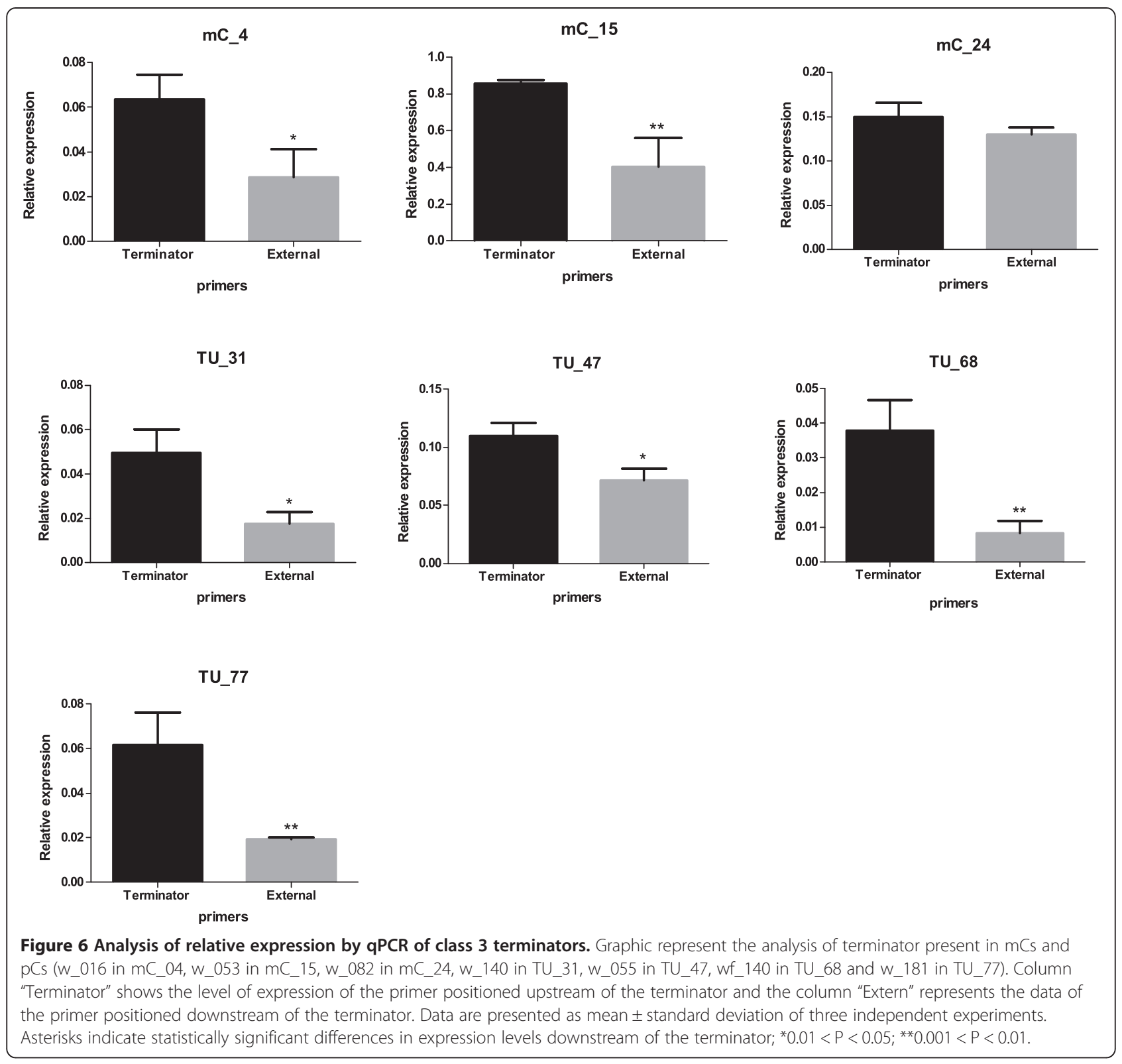

Therefore, to increase the number of putative terminators of the 320 class 1 terminators we lowered the specificity of the class 2 decision rule as follows: class $t_{c 3}-$ class 1 intrinsic terminators validated by only one algorithm, maintaining rules i, iii and iv, described above; and class $t_{\mathrm{c} 4}$ - class 1 intrinsic terminators validated by only one algorithm, maintaining rules $\mathrm{i}$ and iii or iv. Using these new rules the numbers of transcriptional units containing putative terminators analysed in silico increased to $27 \mathrm{mCs}$ and $86 \mathrm{pCs}\left(\mathrm{t}_{\mathrm{c} 2}, \mathrm{t}_{\mathrm{c} 3}\right.$ and $\left.\mathrm{t}_{\mathrm{c} 4}\right)$. Therefore, putative intrinsic terminators have been found, by in silico analysis, in $76 \%$ of transcription units of the $M$. hyopneumoniae genome (82\% of the $33 \mathrm{mCs}$ and in $74 \%$ of the $116 \mathrm{pCs}$ ).
Previous studies have demonstrated the presence of intrinsic terminators in genomes of $M$. genitalium (20\%) and M. pneumoniae (24\%) [16], and also in specific genes such as the 16S-23S rRNA operon of $M$. hyopneumoniae [30], the P1 operon of M. genitalium [31], the MgPa and P65 operons, and the ldh gene of M. pneumoniae [32-34]. Moreover, transcriptional terminators in bacterial genomes belonging to the Firmicutes phylum have also been predicted revealing a high level of conservation [3].

Little information on transcription termination in $M$. hyopneumoniae is available. To validate the in silico putative terminators, experiments were performed for analysis of the transcription end guided by putative terminators. 


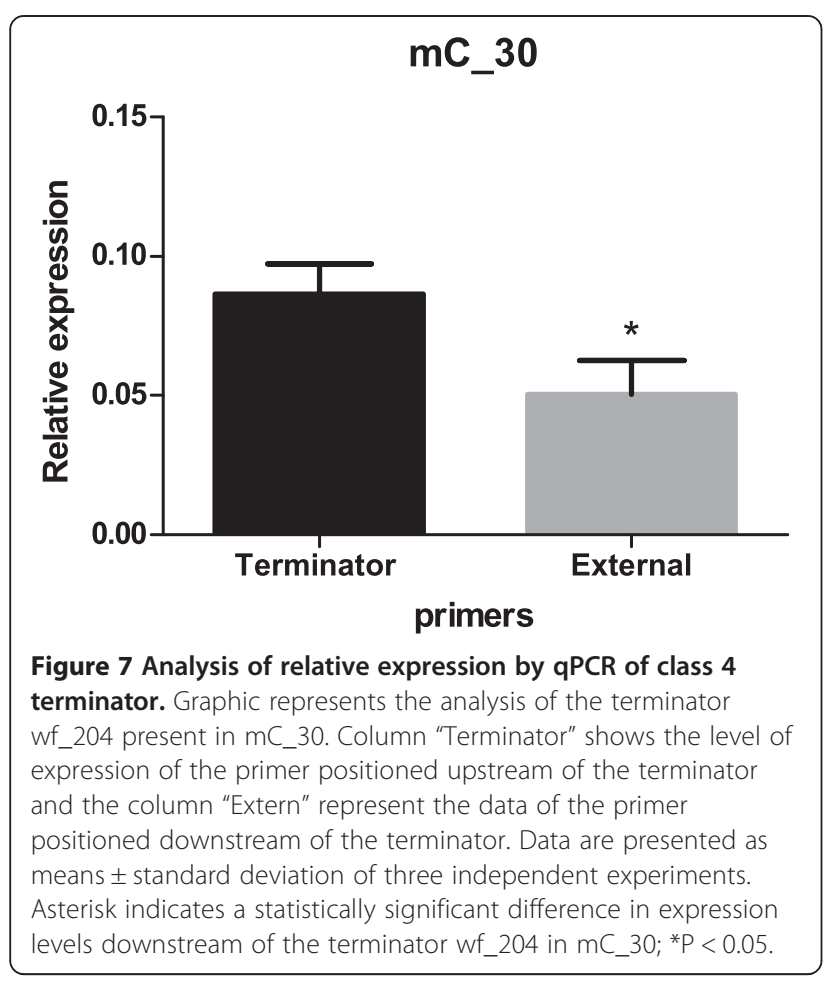

The experimental analyses evaluated 28 of the 113 putative intrinsic terminators belonging to class 2, class 3 and class 4 terminators. The number of terminators experimentally analysed (24\%) was limited due to difficulties in designing primers for some locations as these intergenic regions are characterised by a high adenine and thymine content. In eleven putative terminator sequences, located at the 3 ' end of two mCs and nine pCs, RT-PCR or qPCR demonstrated transcription termination. However, for some intrinsic terminators (12 terminators) transcription continued through the terminator sequence but the level of transcription decreased. The degree of transcriptional change that occurs during quantitative PCR suggests that RNA polymerase continues past the terminator sequence and gradually release the RNA, as demonstrated by transcriptome analysis [10]. As the termination efficiency of an intrinsic terminator is directly related to the stability of the stem-loop structure [4] the relatively high $\Delta G$ values in

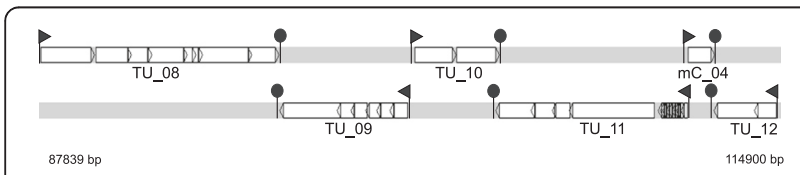

Figure $8 M$. hyopneumoniae genome organisation containing promoter and terminator transcription sites. Figure shows the transcription unit organisation in the genome region from 87,839 to 114,900 base pair. Genome organisation and promoter identification were previously defined $[9,11]$. Black arrows represent promoter sequence and black circle represent terminator sequence. intrinsic terminators could be responsible for the readthrough of some $M$. hyopneumoniae terminator sequences. Supporting our findings, the presence of functional intrinsic terminators was previously demonstrated for genes in other mycoplasma species such as hmw of M. pneumoniae, vmpaU of Mycoplasma agalactiae and for the operon fts $Z$ of $M$. genitalium [18-20].

\section{Conclusion}

In silico predictions, combined with experimental analysis, confirmed the presence of intrinsic terminators in $M$. hyopneumoniae genome. The Figure 8 summarizes the current knowledge related to transcription in $M$. hyopneumoniae. The localisation of terminators sequences at the 3' end of $\mathrm{mCs}$ and $\mathrm{pCs}$ supports previous findings that the $M$. hyopneumoniae genome is organised preferentially in TUs containing two or more genes $[9,10]$. Moreover, the experimental results suggest that at least some terminators have a functional role in mycoplasma. Therefore, the presence of terminator sequences associated with the identification of promoter sequences in $M$. hyopneumoniae transcriptional units ( $\mathrm{mC}$ and $\mathrm{pCs}$ ) [12] suggest that transcription runs on from an upstream promoter and terminates at specific stem-loop regions.

\section{Additional files}

\begin{tabular}{|c|}
\hline $\begin{array}{l}\text { Additional file 1: List of primers used in RT-PCR and qPCR, showing } \\
\text { the sequence, melting point and product size of the primers pairs } \\
\text { used in RT-PCR and qPCR. Amplification efficiency was calculated by } \\
\text { LinRegPCR for primers used in qPCR. }\end{array}$ \\
\hline $\begin{array}{l}\text { Additional file 2: Classes } \mathbf{1}, \mathbf{2}, \mathbf{3} \text { and } \mathbf{4} \text { putative terminators } \\
\text { predicted in } \mathbf{M} \text {. hyopneumoniae. Characteristics of predicted } \\
\text { terminators in the region } 3^{\prime} \text { end after the coding sequence. These } \\
\text { terminators were classified in classes } 1,2,3 \text { and } 4 \text { according to the } \\
\text { criteria described in the text. }\end{array}$ \\
\hline $\begin{array}{l}\text { Additional file 3: Mapping of terminators predicted in } \\
\text { monocistronic units }(\mathbf{m C}) \text { of } \mathbf{M} \text {. hyopneumoniae. Presence of } \\
\text { terminators class } 1 \text { in monocistronic units according the genome } \\
\text { organisation of } M \text {. hyopneumoniae. }\end{array}$ \\
\hline $\begin{array}{l}\text { Additional file 4: Mapping of terminators at the end of } \\
\text { polycistronic units and in internal genes of } \boldsymbol{M} \text {. hyopneumoniae } \\
\text { genome. Presence of terminators class } 1 \text { in polycistronic units according } \\
\text { the genome organisation of } M \text {. hyopneumoniae. }\end{array}$ \\
\hline $\begin{array}{l}\text { Additional file 5: Definition of class } 2 \text { terminators. The scheme } \\
\text { represents a region of } 101600 \text { at } 111600 \text { bp in M. hyopneumoniae } \\
\text { genome with the polycistronic units } 10 \text { (nox) and } 11 \text { (deaA). TU_10 } \\
\text { showed three predicted terminators in the same position; two were } \\
\text { predicted by TransTermHP (red) and one by WebGesTer (yellow). TU_11 } \\
\text { has three predicted terminators in the same position that were predicted } \\
\text { by all three software algorithms. The terminator in orange was predicted } \\
\text { by ARNold. }\end{array}$ \\
\hline
\end{tabular}

\section{Abbreviations}

IR: Intergenic region; CDS: Coding sequences; ORF: Open reading frame; $\mathrm{t}_{\mathrm{c} 1}$ : class 1 terminator; $\mathrm{t}_{\mathrm{c} 2}$ : class 2 terminator; $\mathrm{t}_{\mathrm{c} 3}$ : class 3 terminator; $\mathrm{t}_{\mathrm{c} 4}$ : class 4 terminator; $\triangle \mathrm{G}$ : Gibbs free energy; RT-PCR: Reverse transcriptase PCR; qPCR: quantitative real time PCR; TU: Transcription unit; $\mathrm{PC}$ : polycistronic unit; mC: monocistronic unit; CT: Threshold cycle. 


\section{Competing interests}

The authors declare that they have no competing interests.

\section{Authors' contributions}

TEF, FMS and ISS conceived and designed the experiments. In silico experiment: TEF. RT-PCR and gPCR experiments: TEF and FMS. Data analyse and writing the manuscript: TEF, FMS and ISS. All authors read and approved the final manuscript.

\section{Acknowledgements}

This work was supported by grants from the Brazilian National Research Council (CNPq) and the Fundação de Amparo à Pesquisa do Estado do Rio Grande do Sul (FAPERGS). TEF and FMS received scholarships from Coordenação de Aperfeiçoamento de Pessoal de Nível Superior (CAPES).

Received: 18 December 2014 Accepted: 17 March 2015

\section{Published online: 08 April 2015}

\section{References}

1. Peters JM, Vangeloff AD, Landick R. Bacterial transcription terminators: the RNA 3'-end chronicles. J Mol Biol. 2011;412(5):793-813.

2. d'Aubenton Carafa Y, Brody E, Thermes C. Prediction of rho-independent Escherichia coli transcription terminators. A statistical analysis of their RNA stem-loop structures. J Mol Biol. 1990;216(4):835-58.

3. de Hoon MJ, Makita Y, Nakai K, Miyano S. Prediction of transcriptional terminators in Bacillus subtilis and related species. PLoS Comput Biol. 2005;1(3):e25.

4. Mitra A, Angamuthu K, Jayashree HV, Nagaraja V. Occurrence, divergence and evolution of intrinsic terminators across eubacteria. Genomics. 2009;94(2):110-6.

5. Thacker EL. Diagnosis of Mycoplasma hyopneumoniae. Anim Health Res Rev. 2004:5(2):317-20.

6. Vasconcelos AT, Ferreira HB, Bizarro CV, Bonatto SL, Carvalho MO, Pinto PM, et al. Swine and poultry pathogens: the complete genome sequences of two strains of Mycoplasma hyopneumoniae and a strain of Mycoplasma synoviae. J Bacteriol. 2005;187(16):5568-77.

7. Liu W, Feng Z, Fang L, Zhou Z, Li Q, Li S, et al. Complete genome sequence of Mycoplasma hyopneumoniae strain 168. J Bacteriol. 2011;193(4):1016-7.

8. Siqueira FM, Thompson CE, Virginio VG, Gonchoroski T, Reolon L, Almeida $L G$, et al. New insights on the biology of swine respiratory tract mycoplasmas from a comparative genome analysis. BMC Genomics. 2013;14:175

9. Siqueira FM, Schrank A, Schrank IS. Mycoplasma hyopneumoniae transcription unit organization: genome survey and prediction. DNA Res. 2011;18(6):413-22

10. Siqueira FM, Gerber AL, Guedes RL, Almeida LG, Schrank IS, Vasconcelos AT, et al. Unravelling the transcriptome profile of the Swine respiratory tract mycoplasmas. PLoS One. 2014;9(10):e110327.

11. Weber SS, Sant'Anna FH, Schrank IS. Unveiling Mycoplasma hyopneumoniae promoters: sequence definition and genomic distribution. DNA Research. 2012;19(2):103-15.

12. Siqueira FM, de Souto WS, Cattani AM, Schrank IS. Genome organization in Mycoplasma hyopneumoniae: identification of promoter-like sequences. Mol Biol Rep. 2014;41(8):5395-402.

13. Muto A, Osawa S. The guanine and cytosine content of genomic DNA and bacterial evolution. Proc Natl Acad Sci U S A. 1987:84(1):166-9.

14. Washio T, Sasayama J, Tomita M. Analysis of complete genomes suggests that many prokaryotes do not rely on hairpin formation in transcription termination. Nucleic Acids Res. 1998;26(23):5456-63.

15. Musatovova O, Dhandayuthapani S, Baseman JB. Transcriptional starts for cytadherence-related operons of Mycoplasma genitalium. FEMS Microbiol Lett. 2003;229(1):73-81.

16. Kingsford CL, Ayanbule K, Salzberg SL. Rapid, accurate, computational discovery of Rho-independent transcription terminators illuminates their relationship to DNA uptake. Genome Biol. 2007;8(2):R22.

17. Mitra A, Kesarwani AK, Pal D, Nagaraja V. WebGeSTer DB-a transcription terminator database. Nucleic Acids Res. 2011;39(Database issue):D129-35.

18. Waldo RH, Popham PL, Romero-Arroyo CE, Mothershed EA, Lee KK, Krause DC. Transcriptional analysis of the hmw gene cluster of Mycoplasma pneumoniae. J Bacteriol. 1999;181(16):4978-85.
19. Benders GA, Powell BC, Hutchison CA. Transcriptional analysis of the conserved ftsZ gene cluster in Mycoplasma genitalium and Mycoplasma pneumoniae. J Bacteriol. 2005;187(13):4542-51.

20. Chopra-Dewasthaly R, Citti C, Glew MD, Zimmermann M, Rosengarten R, Jechlinger W. Phase-locked mutants of Mycoplasma agalactiae: defining the molecular switch of high-frequency Vpma antigenic variation. Mol Microbiol. 2008;67(6):1196-210.

21. Naville M, Ghuillot-Gaudeffroy A, Marchais A, Gautheret D. ARNold: a web tool for the prediction of Rho-independent transcription terminators. RNA Biol. 2011;8(1):11-3.

22. Rutherford K, Parkhill J, Crook J, Horsnell T, Rice P, Rajandream MA, et al. Artemis: sequence visualization and annotation. Bioinformatics. 2000;16(10):944-5.

23. Friis NF. Some recommendations concerning primary isolation of Mycoplasma suipneumoniae and Mycoplasma flocculare a survey. Nordisk Veterinaer Medicin. 1975;27(6):337-9.

24. Ruijter JM, Ramakers C, Hoogaars WM, Karlen Y, Bakker O, van den Hoff MJ, et al. Amplification efficiency: linking baseline and bias in the analysis of quantitative PCR data. Nucleic Acids Res. 2009;37(6):e45.

25. Livak KJ, Schmittgen TD. Analysis of relative gene expression data using real-time quantitative $P C R$ and the 2(-Delta Delta $C(T)$ ) Method. Methods. 2001;25(4):402-8.

26. Madsen ML, Puttamreddy S, Thacker EL, Carruthers MD, Minion FC. Transcriptome changes in Mycoplasma hyopneumoniae during infection. Infect Immun. 2008;76(2):658-63.

27. Arrebola E, Carrión VJ, Cazorla FM, Pérez-García A, Murillo J, de Vicente A. Characterisation of the mgo operon in Pseudomonas syringae pv. syringae UMAF0158 that is required for mangotoxin production. BMC Microbiol. 2012;12:10.

28. Unniraman S, Prakash $R$, Nagaraja V. Alternate paradigm for intrinsic transcription termination in eubacteria. J Biol Chem. 2001;276(45):41850-5.

29. Ingham CJ, Hunter IS, Smith MC. Rho-independent terminators without 3 ' poly- $U$ tails from the early region of actinophage $\varnothing C 31$. Nucleic Acids Res. 1995;23(3):370-6.

30. Taschke $C$, Herrmann R. Analysis of transcription and processing signals of the 16S-23S rRNA operon of Mycoplasma hyopneumoniae. Mol Gen Genet. 1986;205(3):434-41.

31. Inamine JM, Loechel S, Hu PC. Analysis of the nucleotide sequence of the P1 operon of Mycoplasma pneumoniae. Gene. 1988;73(1):175-83.

32. Inamine JM, Loechel S, Collier AM, Barile MF, Hu PC. Nucleotide sequence of the $\mathrm{MgPa}$ (mgp) operon of Mycoplasma genitalium and comparison to the P1 (mpp) operon of Mycoplasma pneumoniae. Gene. 1989;82(2):259-67.

33. Krause DC, Proft $T$, Hedreyda $C T$, Hilbert $H$, Plagens $H$, Herrmann $R$. Transposon mutagenesis reinforces the correlation between Mycoplasma pneumoniae cytoskeletal protein HMW2 and cytadherence. J Bacteriol. 1997;179(8):2668-77.

34. Halbedel S, Stülke J. Tools for the genetic analysis of Mycoplasma. Int J Med Microbiol. 2007;297(1):37-44.

\section{Submit your next manuscript to BioMed Central and take full advantage of:}

- Convenient online submission

- Thorough peer review

- No space constraints or color figure charges

- Immediate publication on acceptance

- Inclusion in PubMed, CAS, Scopus and Google Scholar

- Research which is freely available for redistribution 